INPLASY

PROTOCOL

To cite: Armstrong et al. The association between youth blood pressure and adult coronary artery calcification: A systematic review. Inplasy protocol 2021120015. doi: 10.37766/inplasy2021.12.0015

Received: 02 December 2021

Published: 02 December 2021

Corresponding author: Costan Magnussen

costan.magnussen@utas.edu.au

Author Affiliation:

University of Tasmania.

Support: None.

Review Stage at time of this submission: Preliminary searches.

Conflicts of interest:

None declared.

\section{The association between youth blood pressure and adult coronary artery calcification: A systematic review}

Armstrong, MK¹; Sun, J2; Wu, F3; Xi, B4; Magnussen, CG55.

Review question / Objective: The aim of this systematic review is to determine the relationship between youth (children or adolescents) blood pressure (BP) and coronary artery calcification (CAC) measured in adulthood.

Condition being studied: Systolic BP levels (exposure) and CAC scores (outcome) among population-based samples. Information sources: Medline via PubMed, Web of Science, and Embase databases will be searched from inception to October 2021. Reference lists of eligible studies will be used to identify additional studies that may have been missed by the database search. Finally, potentially eligible studies may be identified through consultation with experts in the field.

INPLASY registration number: This protocol was registered with the International Platform of Registered Systematic Review and Meta-Analysis Protocols (INPLASY) on 02 December 2021 and was last updated on 02 December 2021 (registration number INPLASY2021120015).

\section{INTRODUCTION}

Review question / Objective: The aim of this systematic review is to determine the relationship between youth (children or adolescents) blood pressure (BP) and coronary artery calcification (CAC) measured in adulthood.

Rationale: The importance of early life exposure to cardiovascular risk factors for cardiovascular health is well established but also underappreciated in the clinical setting. A recent systematic review and meta-analysis observed that elevated BP in childhood or adolescence was associated with intermediate cardiovascular endpoints in adulthood, including elevated pulse wave velocity, carotid intima-media thickness, and left ventricular hypertrophy. Nevertheless, the association between youth BP and adult CAC, a robust 
intermediate cardiovascular end point, remains unclear. The relationship between youth BP and adult CAC is important as CAC quantifies the atherosclerotic burden in the coronary arteries, the primary site of ischemic heart disease. Therefore, this study will seek to clarify the association between youth systolic BP and adult CAC via systematic review and identify areas for future research.

Condition being studied: Systolic BP levels (exposure) and CAC scores (outcome) among population-based samples.

\section{METHODS}

Search strategy: Three databases, Medline (via PubMed), Web of Science, and Embase, will be searched from inception to October 2021 using the following search string: (("blood pressure" or "arterial pressure" or "hypertension") and ("child" or "children" or "adolescent" or "adolescents" or "adolescence" or "childhood" or "students" or "youth" or "teenagers" or "teens" or "pediatric" or "pediatrics")) and (("coronary artery calcium" or "coronary artery calcification") and ("adult" or "adults" or "adulthood")) and ("cohort" or "Iongitudinal" or "follow-up" or "prospective"). Additional studies will be identified through a search of the reference lists of eligible studies and via consultation with experts in the field (CGM and BX). Title and abstract screening of identified studies and subsequent full text review of eligible studies will be performed by MKA and JS with disagreements resolved by consensus or consultation with an expert (CGM).

Participant or population: Populationbased sample. Youth defined as individuals aged $\leq 18$ years at baseline and adult defined as individuals aged $>18$ years at follow-up.

Intervention: None.

\section{Comparator: None.}

Study designs to be included: Cohort studies.
Eligibility criteria: Human cohort studies published in English with measures of youth (individuals aged $\leq 18$ years) BP and follow-up measures of adult (individuals aged $>18$ years) CAC. Only, full text, original empirical research journal articles are considered and studies among individuals with known disease or special interest groups are not eligible for inclusion.

Information sources: Medline via PubMed, Web of Science, and Embase databases will be searched from inception to October 2021. Reference lists of eligible studies will be used to identify additional studies that may have been missed by the database search. Finally, potentially eligible studies may be identified through consultation with experts in the field.

Main outcome(s): CAC score measured in adulthood via computed tomography.

Data management: Relevant data will be collected from eligible studies using a predetermined data extraction table which will include details on: study, first author, year of publication, country of study, sample size, proportion of males, duration of follow-up, age at baseline, age at followup, prevalence of CAC, measurement of youth BP, definition of CAC, findings, and covariates.

Quality assessment / Risk of bias analysis: Study quality will be assessed according to the Newcastle-Ottawa Quality assessment scale for cohort studies.

Strategy of data synthesis: Qualitative only.

Subgroup analysis: None.

Sensitivity analysis: None.

Language: English.

Country(ies) involved: United States, Australia, and China.

Keywords: child, adolescent, heart diseases, cohort studies, atherosclerosis, prospective studies. 
Contributions of each author:

Author 1 - Matthew Armstrong Conception, design, methodology, data search, data curation, manuscript preparation.

Email: mkrmstrong@uiowa.edu

Author 2 - Jiahong Sun - Methodology, data search, data curation, manuscript revision. Author 3 - Feitong Wu - Methodology, manuscript revision.

Author 4 - Bo Xi - Methodology, manuscript revision.

Author 5 - Costan Magnussen Conception, design, methodology, manuscript revision. 